\title{
Montanide ISA 51 VG
}

National Cancer Institute

\section{Source}

National Cancer Institute. Montanide ISA 51 VG. NCI Thesaurus. Code C84843.

An water-in-oil (w/o) emulsion with immunomoadjuvant activity. Montanide ISA 51 VG appears to act by enhancing the immune system's cytotoxic T-lymphocyte (CTL) response against antigen(s) in vaccines. The surfactant mannide monooleate in Montanide ISA 51 VG contains vegetable-grade (VG) oleic acid derived from olive oil. 